International Mathematical Forum, 2, 2007, no. 11, 515 - 524

\title{
Compatible Mappings of Type $(A-1)$ and Type $(A-2)$ and Common Fixed Points in Fuzzy Metric Spaces
}

\author{
M. S. Khan ${ }^{1}$ \\ Department of Mathematics and Statistics \\ Sultan Qaboos University \\ P.O. Box 36, P. C. 123 Al-Khod, Sultanate of Oman \\ H. K. Pathak \\ Department of Mathematics, Kalyan Vidyalaya \\ Bhilainagar (C. G.) 490006 India \\ Reny George \\ Department of Mathematics \\ St. Thomas College \\ Bhilai (C. G. ) 490006 India
}

\begin{abstract}
The purpose of this paper is to obtain common fixed point theorems for compatible maps of type (A-I) and type (A2) on fuzzy metric spaces. Our results extend, generalize and fuzzify several fixed point theorems on metric _paces, menger probabilistic metric spaces, uniform spaces and fuzzy metric spaces.
\end{abstract}

\section{Mathematics Subject Classification: 47H10, 54H25}

\section{INTRODUCTION}

Zadeh's introduction [25] of the notion of fuzzy sets laid down the foundation of fuzzy mathematics. In the last two decades there were a tremendous development and growth in fuzzy mathematics ([1], [3], [5], [6], [12], [19]). In [6] Grabiec extended the well known fixed-point theorems of Banach [2] and Edelsten [4] to fuzzy metric spaces in the sense of Kramosil and Michalek [12]. Moreover it appears that the study of Kramosil and Michalek [12] of fuzzy metric space paves the way for developing soothing machinery in the field of fixed-point theorems, in particular, for the study of contractive type maps. In

\footnotetext{
${ }^{1}$ Corresponding author
} 
[5] Fang proved some fixed point theorems in fuzzy metric spaces, which improve, generalize, extends and unify some main results of [2], [4], [8] and [17]. Following Grabiec [6] and Kramosil-Michalek [12], Mishra-Sharma-Singh [13] obtained common fixed point theorems for compatible maps and asymptotically commuting maps on fuzzy metric spaces, which generalize, extend, and fuzzify several fixed point theorems for contractive type maps on metric spaces and other spaces.

In this paper we introduce the concept of compatible mappings of type (A1) and type (A-2) in fuzzy metric space and show that they are equivalent to compatible mappings under certain conditions. In the Sequel we prove some common fixed point theorem for compatible mappings of type (A-1) and type (A-2) on fuzzy metric spaces which generalize, extend, unify and fuzzify several well known fixed point theorems for contractive type maps on metric spaces, Menger spaces, uniform spaces and fuzzy metric spaces.

\section{Preliminaries}

Following Grabiec [6] and Kramosil-Michalek [12] we have the following notations and definitions.

A fuzzy metric space (Shortly an FM space) is an ordered triplet $(X, M, *)$ consisting of a nonempty set $X$, a fuzzy set $M$ in $X^{2} \times[0, \infty)$ and a continuous $T$-norm*. The functions $M(x, y,):.[0, \infty) \rightarrow[0$,$] are left continuous and are$ assumed to satisfy the following conditions:

$$
(F M-1) \ldots \ldots . \quad M(x, y, t)=1
$$

for all $t>0$ if and only if $x=y$.

$$
\begin{array}{rlrl}
(F M-2) & \ldots \ldots & M(x, y, 0) & =0 . \\
(F M-3) & \ldots \ldots . . & M(x, y, t) & =M(y, x, t) . \\
(F M-4) \ldots & M(x, y, t)^{*} M(y, z, s) & <M(x, z, t+s)
\end{array}
$$

for all $x, y, z$ in $X$ and $t, s>=0$.

Gragiec [6] has shown that $M(x, y,$.$) is nondecreasing for all x, y$ in $X$.

In all that follows $N$ denotes the set of natural numbers and $X$ an $F M$ space $\left(X, M,{ }^{*}\right)$ with the following condition:

$$
(F M-5) \ldots \ldots . . \lim _{t->\infty} M(x, y, t)=1 \text { for all } x, y \text { in } X .
$$

The proof of the following lemma is given in [6].

Lemma 2.1. Let $\left\{y_{n}\right\}$ be a sequence in an FM-space $X$. If there exists a positive number $k<1$ such that

$$
M\left(y_{n+2}, y_{n+1}, k t\right) \geq M\left(Y_{n+1}, y_{n}, t\right), t>0, n \in N
$$

then $\left\{y_{n}\right\}$ is a Cauchy sequence in $X$.

From $(F M-5)$, the following lemma follows immediately: 
Lemma 2.2. If for two points $x, y$ in $X$ and a positive number $k<1$

$$
M(x, y, k t) \geq M(x, y, t) \text { then } x=y .
$$

3. Compatible Maps of Type (A-1) and Type (A-2)

In this section we show that compatible mappings of type (A-1) and type (A2 ) in fuzzy metric space are equivalent to compatible mappings under certain conditions.

Definition 3.1. Let $S$ and $T$ be self maps of an $F M$-space $X$. The mappings $S$ and $T$ are said to be compatible if $\lim _{n \rightarrow \infty} M\left(S T x_{n}, T S x_{n}, t\right)=1$, whenever $\left\{x_{n}\right\}$ is a sequence in $X$ such that $\lim _{n \rightarrow \infty} S x_{n}=\lim _{n \rightarrow>\infty} T x_{n}=z$ for some $z$ in $X$.

Definition 3.2. Let $S$ and $T$ be self maps of an $F M$-space $X$. The mappings $S$ and $T$ are said to be compatible of type $(A)$ if $\lim _{n->\infty} M\left(S T x_{n}, T T x_{n}, t\right)=$ 1 and $\lim _{n \rightarrow>\infty} M\left(T S x_{n}, S S x_{n}, t\right)=1$, whenever $\{x n\}$ is a sequence in $X$ such that $\lim _{n \rightarrow>\infty} S x_{n}=\lim _{n \rightarrow>\infty} T x_{n}=z$ for some $z$ in $X$.

Definition 3.3. Let $S$ and $T$ be self maps of an FM-space $X$. The pair of mappings $(S, T)$ is said to be compatible of type $(A-1)$ if

$\lim _{n \rightarrow>\infty} M\left(S T x_{n}, T T x_{n}, t\right)=1$, whenever $\left\{x_{n}\right\}$ is a sequence in $X$ such that $\lim _{n \rightarrow \infty} S x_{n}=\lim _{n \rightarrow>\infty} T x_{n}=z$ for some $z$ in $X$.

Definition 3.4. Let $S$ and $T$ be self maps of an FM-space $X$. The pair of mappings $(S, T)$ is said to be compatible of type $(A-2)$ if

$\lim _{n \rightarrow>\infty} M\left(T S x_{n}, S S x_{n}, t\right)=1$, whenever $\left\{x_{n}\right\}$ is a sequence in $X$ such that $\lim _{n \rightarrow \infty} S x_{n}=\lim _{n \rightarrow \infty} T x_{n}=z$ for some $z$ in $X$.

Clearly, if a pair of mappings $(S, T)$ is compatible of type $(A-1)$ then the pair $(T, S)$ is compatible of type $(A-2)$. Further from the definitions it is clear that if $S$ and $T$ are compatible mappings of type $(A)$ then the pair $(S, T)$ is compatible of type $(A-1)$ as well as type $(A-2)$.

We now cite the following propositions which gives the condition under which the definitions 3.1, 3.3 and 3.4 becomes equivalent.

Proposition 3.5. Let $S$ and $T$ be self maps of an $F M$-space $X$.

a) If $T$ is continuous then the pair of mappings $(S, T)$ is compatible of type $(A-1)$ iff $S$ and $T$ are compatible.

b) If $S$ is continuous then the pair $(S, T)$ is compatible of type $(A-2)$ iff $S$ and $T$ are continuous.

Proof. a) Let $\lim _{n \rightarrow>\infty} S x_{n}=\lim _{n \rightarrow>\infty} T x_{n}=z$ for some $z$ in $X$, and let the pair $(S, T)$ be compatible of type $(A-I)$. Since $T$ is continuous we have $\lim _{n \rightarrow>\infty} T S x_{n}=T z$ and $\lim _{n \rightarrow \infty} T T x_{n}=T z$. 
Therefore it follows that $M\left(S T x_{n}, T S x_{n}, t\right) \geq M\left(S T x_{n}, T T x_{n}, t / 2\right)^{*}$

$M\left(T T x_{n}, T S x_{n}, t / 2\right)$ yields $\lim _{n \rightarrow \infty} M\left(S T x_{n}, T S x_{n}, t\right) \geq 1^{*} 1=1$ and so the mappings $S$ and $T$ are compatible.

Now let $S$ and $T$ be compatible.

Therefore it follows that $M\left(S T x_{n}, T T x_{n}, t\right) \geq M\left(S T x_{n}, T S x_{n}, t / 2\right)^{*}$ $M\left(T S x_{n}, T T x_{n}, t / 2\right)$ yields $\lim _{n \rightarrow \infty} M\left(S T x_{n}, T T x_{n}, t\right) \geq 1^{*} 1=1$ and so the pair of mappings $(S, T)$ are compatible of type $(A-1)$

b) Let $\lim _{n \rightarrow \infty} S x_{n}=\lim _{n \rightarrow>\infty} T x_{n}=z$ for some $z$ in $X$, and let the pair $(S, T)$ be compatible of type $(A-2)$. Since $S$ is continuous we have $\lim _{n \rightarrow \infty} S T x_{n}=S z$ and $\lim _{n \rightarrow \infty} S S x_{n}=S z$.

It follows that $M\left(S T x_{n}, T S x_{n}, t\right) \geq M\left(S T x_{n}, S S x_{n}, t / 2\right)^{*}$ $M\left(S S x_{n}, T s x_{n}, t / 2\right)$ yields $\lim _{n \rightarrow \infty} M\left(S T x_{n}, T S x_{n}, t\right) \geq 1^{*} 1=1$ and so the mappings $S$ and $T$ are compatible.

Now let $S$ and $T$ be compatible. Then we have $M\left(T S x_{n}, S S x_{n}, t\right) \geq$ $M\left(T S x_{n}, S T x_{n}, t / 2\right)^{*} M\left(S T X_{n}, S S x_{n}, t / 2\right)$ yields $\lim _{n \rightarrow \infty} M\left(T S x_{n}, S S x_{n}, t\right)=$ $1^{*} 1=1$ and so the pair $(S, T)$ are compatible of type $(A-2)$.

Next we give some properties of compatible mappings of type $(A-1)$ and type $(A-2)$ which will be used in our main theorem.

Proposition 3.6. Let $S$ and $T$ be self maps of an FM space $X$. If the pair $(S, T)$ are compatible of type $(A-I)$ and $S z=T z$ for some $z$ in $X$ then $S T z=T T z$.

Proof. Let $\left\{x_{n}\right\}$ be a sequence in $X$ defined by $x_{n}=z$ for $n=1,2, \ldots$ and let $T z=S z$. Then we have $\lim _{n \rightarrow \infty} S x_{n}=S z$ and $\lim _{n \rightarrow \infty} T x_{n}=T z$. Since the pair $(S, T)$ is compatible of type $(A-1)$ we have $M(S T z, T t z, t)=$ $\lim _{n \rightarrow>\infty} M\left(S T x_{n}, T T x_{n}, t\right)=1$. Hence $S T z=T T z$.

Proposition 3.7. Let $S$ and $T$ be self maps of an FM-space $X$. If the pair $(S, T)$ is compatible of type $(A-2)$ and $S z=T z$ for some $z$ in $X$ then $T S z=S S z$.

Proof. Let $\left\{x_{n}\right\}$ be a sequence in $X$ defined by $x_{n}=z$ for $n=1,2, \ldots$ and let $T z=S z$. Then we have $\lim _{n \rightarrow \infty} S x_{n}=S z$ and $\lim _{n \rightarrow \infty} T x_{n}=T z$. Since the pair $(S, T)$ is compatible of type $(A-2)$ we have $M(T S z, S S z, t)=$ $\lim _{n \rightarrow>\infty} M\left(T S x_{n}, S S x_{n}, t\right)=1$. Hence $T S z=S S z$.

Proposition 3.8. Let $S$ and $T$ be self maps of an $F M$-space $X$ with $t^{*} t>t$ for all $t$ in $[0,1]$. If the pair $(S, T)$ are compatible of type $(A-1)$ and $S x_{n}, T x_{n} \rightarrow z$ for some $z$ in $X$ and a sequence $\left\{x_{n}\right\}$ in $X$ then, $T T x_{n} \rightarrow S z$ if $S$ is continuous at $z$. 
Proof. Since $S$ is continuous at $Z$ we have $S T x_{n} \rightarrow S z$. Since the pair $(S, T)$ are compatible of type $(A-1)$, we have $M\left(S T x_{n}, T T x_{n}, t\right) \rightarrow 1$ as $n->\infty$. It follows that $M\left(S z, T T x_{n}, t\right) \geq M\left(S z, S T x_{n}, t / 2\right)^{*} M$ $\left(S T x_{n}, T T x_{n}, t / 2\right)$ yields $\lim _{n->\infty} M\left(S z, T T x_{n}, t\right) \geq 1^{*} 1=1$ and so we have $T T x_{n} \rightarrow S z$ as $n->\infty$.

Proposition 3.9. Let $S$ and $T$ be self maps of an $F M$-space $X$ with $t^{*} t \geq t$ for $t$ in $[0,1]$. If the pair $(S, T)$ are compatible of type $(A-2)$ and $S x_{n}, T x_{n} \rightarrow z$ for some $z$ in $X$ and a sequence $\left\{x_{n}\right\}$ in $X$ then, $S S x_{n} \rightarrow T z$ if $T$ is continuous at $z$.

Proof. Since $T$ is continuous at $Z$ we have $T S x_{n} \rightarrow T z$. Since the pair $(S, T)$ are compatible of type $(A-1)$, we have $M\left(T S x_{n}, S S x_{n}, t\right) \rightarrow 1$ as $n->\infty$. It follows that $M\left(T z, S S x_{n}, t\right) \geq M\left(T z, T S x_{n}, t / 2\right)^{*} M$ $\left(T S x_{n}, S S x_{n}, t / 2\right)$ yields $\lim _{n \rightarrow \infty} \bar{M}\left(T z, S S x_{n}, t\right) \geq 1 * 1=1$ and so we have $S S x_{n} \rightarrow T z$ as $n->\infty$.

\section{Main Results}

Let $\left(X, M,{ }^{*}\right)$ be an $F M$-space with $t^{*} t \geq 1$ for all $\mathrm{t}$ in $[0,1]$ and $P, Q, S, T$ be self maps of $X$ such that

$$
P(X) \subseteq T(X) \text { and } Q(X) \subseteq S(X)
$$

$$
\begin{gathered}
\geq p\left[M(P x, S x, k t)^{*} M(Q y, T y, k t)+M(P x, T y, k t)^{*} M(Q y, S x, k t)\right]+M \\
(S x, T y, t)^{*} M(P x, S x, t)^{*} M(Q y, T y, t)^{*} M(P x, T y, \alpha t)^{*} M(Q y, S x,(2-\alpha) t)
\end{gathered}
$$

for all $x, y$ in $X, p \geq 0, t>0$ and $\alpha \in(0,2)$ and $k \in(0,1)$.

For some arbitrary $x_{0}$ in $X$, by (4.1) we choose $x_{1}$ in $X$ such that $P x_{0}=T x_{1}$, and for this $x_{1}$ there exists $x_{2}$ such that $S x_{2}=Q x_{1}$. Continuing this process we define the sequence $\left\{y_{n}\right\}$ in $X$ such that

$$
y_{2 n}=P x_{2 n}=T x_{2 n+1} \text { and } y_{2 n+1}=Q x_{2 n+1}=S x_{2 n+2}
$$

Lemma 4.1. Let $P, Q, S$ and $T$ be self maps of an $F M$-space $\left(X, M,{ }^{*}\right)$ with $t^{*} t \geq t$ for all $t$ in $[0,1]$ satisfying (4.1) and (4,2). Then the sequence $\left\{y_{n}\right\}$ defined by (4.3) is a Cauchy sequence. 
Proof. By (4.2) for $\alpha=1+q, q \in(0,1)$ we have,

$$
\begin{aligned}
& {\left[1+p \cdot M\left(y_{2 n} y_{2 n+1}, k t\right)\right]^{*} M\left(y_{2 n+1}, y_{2 n+2}, k t\right)} \\
& =\left[1+p \cdot M\left(T x_{2 n+1}, S x_{2 n+2}, k t\right)\right]^{*} M\left(Q x_{2 n+1}, P x_{2 n+2}, k t\right) \\
& \geq p\left[M\left(P x_{2 n+2}, S x_{2 n+2}, k t\right)^{*} M\left(Q x_{2 n+1}, T x_{2 n+1}, k t\right)\right. \\
& \left.+M\left(P x_{2 n+2}, T x_{2 n+1}, k t\right)^{*} M\left(Q x_{2 n+1}, S x_{2 n+2}, k t\right)\right] \\
& \left.+M S x_{2 n+2}, T x_{2 n+1} y, t\right)^{*} M\left(P x_{2 n+2}, S x_{2 n+2}, t\right)^{*} M\left(Q x_{2 n+1}, T x_{2 n+1}, t\right)^{*} \\
& M\left(P x_{2 n+2}, T x_{2 n+1},(1+q) t\right)^{*} M\left(Q x_{2 n+1}, S x_{2 n+2},(1-q) t\right) \\
& =p\left[M\left(y_{2 n+2}, y_{2 n+1}, k t\right)^{*} M\left(y_{2 n+1}, y_{2 n}, k t\right)+M\left(y_{2 n+2}, y_{2 n}, k t\right)^{*} M\left(y_{2 n+1}, y_{2 n+1}, k t\right)\right] \\
& +M\left(y_{2 n+1}, y_{2 n}, t\right)^{*} M\left(y_{2 n+2}, y_{2 n+1}, t\right)^{*} M\left(y_{2 n+1}, y_{2 n}, t\right)^{*} \\
& M\left(y_{2 n+2}, y_{2 n},(1+q) t\right)^{*} M\left(y_{2 n+1}, y_{2 n+1},(1-q) t\right) \\
& =p\left[M\left(y_{2 n+2}, y_{2 n+1}, k t\right)^{*} M\left(y_{2 n+1}, y_{2 n}, k t\right)+M\left(y_{2 n+2}, y_{2 n}, k t\right)^{*} 1\right] \\
& +M\left(y_{2 n+1}, y_{2 n}, t\right)^{*} M\left(y_{2 n+2}, y_{2 n+1}, t\right)^{*} M\left(y_{2 n+1}, y_{2 n}, t\right)^{*} M\left(y_{2 n+2}, y_{2 n},(1+q) t\right)^{*} 1 \\
& \geq p\left[M\left(y_{2 n+2}, y_{2 n+1}, k t\right)^{*} M\left(y_{2 n+1}, y_{2 n}, k t\right)\right]+M\left(y_{2 n+1}, y_{2 n}, t\right)^{*} \\
& M\left(y_{2 n+2}, y_{2 n+1}, t\right)^{*} M\left(y_{2 n+1}, y_{2 n}, t\right)^{*} M\left(y_{2 n}, y_{2 n+1}, q t\right)^{*} M\left(y_{2 n+1}, y_{2 n+2}, t\right)^{*} 1 \\
& \geq p\left[M\left(y_{2 n+2}, y_{2 n+1}, k t\right)^{*} M\left(y_{2 n+1}, y_{2 n}, k t\right)\right]+M\left(y_{2 n+1}, y_{2 n}, t\right)^{*} \\
& M\left(y_{2 n+2}, y_{2 n+1}, t\right)^{*} M\left(y_{2 n}, y_{2 n+1}, q t\right) .
\end{aligned}
$$

Thus it follows that

$$
M\left(y_{2 n+1}, y_{2 n+2}, k t\right) \geq M\left(y_{2 n+1}, y_{2 n}, t\right)^{*} M\left(y_{2 n+2}, y_{2 n+1}, t\right)^{*} M\left(y_{2 n}, y_{2 n+1}, q t\right)
$$

Since the $t$-norm * is continuous and $M(x, y)$ is left continuous, letting $q \rightarrow 1$, we have $M\left(y_{2 n+1}, y_{2 n+2}, k t\right) \geq M\left(y_{2 n}, y_{2 n+1}, t\right)^{*} M\left(y_{2 n+1}, y_{2 n+2}, t\right)$.

Similarly we get $M\left(y_{2 n+2}, y_{2 n+3}, k t\right) \geq M\left(y_{2 n+1}, y_{2 n+2}, t\right)^{*} M\left(y_{2 n+2}, y_{2 n+3}, t\right)$. In general we have $M\left(y_{m+1}, y_{m+2}, k t\right) \geq M\left(y_{m}, y_{m+1}\right)^{*} M\left(y_{m+1}, y_{m+2}, t\right)$.

Consequently it follows that

$$
M\left(y_{m+1}, y_{m+2}, k t\right) \geq M\left(y_{m}, y_{m+1}, t\right)^{*} M\left(y_{m+1}, y_{m+2}, t / k^{p}\right), m, p \in N
$$

By noting that $M\left(y_{m+1}, y_{m+2}, t / k^{p}\right) \rightarrow 1$ as $n \rightarrow \infty$, we have $M\left(y_{m+1}, y_{m+2}, k t\right) \geq$ $M\left(y_{m}, y_{m+1}, t\right)$. Hence by Lemma $2.1\left\{y_{n}\right\}$ is a Cauchy sequence.

Theorem 4.2. Let $\left(X, M,^{*}\right)$ be a complete $F M$-space with $t^{*} t \geq t, t \in[0,1]$, and let $P, Q, S$ and $T$ be self maps of $X$ satisfying (4.1),(4.2) and the following:

the pairs $(P, S)$ and $(Q, T)$ are compatible of type $(A-1)$ or type $(A-2)$

one of $S$ and $T$ is continuous

Then $P, Q, S$ and $T$ have a unique common fixed point in $X$. 
Proof. Let the pairs $(P, S)$ and $(Q, T)$ be compatible of type $(A-1)$ and suppose $T$ is continuous. By Lemma 2.1 the sequence $\left\{y_{n}\right\}$ defined by (4.3) is a Cauchy sequence in $X$ and since $X$ is complete it should converge to some point say $z$ in $x$. Consequently the subsequences $\left\{P x_{n}\right\},\left\{T x_{2 n+1}\right\},\left\{Q x_{2 n+1}\right\}$ and $\left\{S x_{2 n+2}\right\}$ also converge to the same point z. Since $T$ is continuous $T T x_{2 n+1} \rightarrow$ $T z$ as $n \rightarrow \infty$, also since the pair $(Q, T)$ are compatible of type $(A-1)$, by proposition (3.4) we have $Q T x_{2 n+1} \rightarrow T z$ as $n \rightarrow \infty$.

Now for $\alpha=1,(4.2)$ yields,

$$
\begin{aligned}
& {\left[1+p \cdot M\left(S x_{2 n}, T T x_{2 n+1}, k t\right)\right]^{*} M\left(P x_{2 n}, Q T x_{2 n+1}, k t\right)} \\
& \geq p\left[M\left(P x_{2 n}, S x_{2 n}, k t\right)^{*} M\left(Q T x_{2 n+1}, T T x_{2 n+1}, k t\right)+M\left(P x_{2 n}, T T x_{2 n+l}, k t\right)\right. \\
& \left.{ }^{*} M\left(Q T x_{2 n+1}, S x_{2 n}, k t\right)\right]+M\left(S x_{2 n}, T T x_{2 n+1}, t\right)^{*} M\left(p x_{2 n}, S x_{2 n}, t\right) \\
& { }^{*} M\left(Q T x_{2 n+1}, T T x_{2 n+1}, t\right)^{*} M\left(P x_{2 n}, T T x_{2 n+1}, t\right)^{*} M\left(Q T x_{2 n+1}, S x_{2 n}, t\right)
\end{aligned}
$$

which gives $M(z, T z, k t) \geq M(z, T z, t)^{*} 1^{*} 1^{*} M(z, T z, t)^{*} M(T z, z, t)$. This shows that $T z=z$. Again by (4.2) with $\alpha=1$, we have

$$
\begin{aligned}
& {\left[1+p \cdot M\left(S x_{2 n}, T z, k t\right)\right]^{*} M\left(P x_{2 n}, Q z, k t\right)} \\
& \geq p\left[M\left(P x_{2 n}, S x_{2 n}, k t\right)^{*} M(Q z, T z, k t)+M\left(P x_{2 n}, T z, k t\right)^{*} M\left(Q z, S x_{2 n}, k t\right)\right] \\
& +M\left(S x_{2 n}, T z, t\right)^{*} M\left(P x_{2 n}, S x_{2 n}, t\right)^{*} M(Q z, T z, t)^{*} M\left(P x_{2 n}, T z, t\right)^{*} M\left(Q z, S x_{2 n}, t\right)
\end{aligned}
$$

which yields $M(z, Q z, k t) \geq 1^{*} 1^{*} M(Q z, z, t)^{*} 1^{*} 1^{*} M(Q z, z, t)$ and so $Q z=z$. Hence by (4.1) there exists some $u$ in $X$ such that $Q z=S u$. By (4.2) with $\alpha=1$ we have,

$$
\begin{aligned}
& {[1+p \cdot M(S u, T z, k t)]^{*} M(P u, Q z . k t)} \\
& \quad \geq p\left[M(P u, S u, k t)^{*} M(Q z, T z, k t)+M(P u, T z, k t)^{*} M(Q z, S u, k t)\right] \\
& +M(S u, T z, t)^{*} M(P u, S u, t)^{*} M(Q z, T z, t)^{*} M(P u, T z, t)^{*} M(Q z, S u, t)
\end{aligned}
$$

which yields $(P u, z, k t) \geq 1^{*} M(p u, z, t)^{*} 1^{*} M(P u, z, t)^{*} 1$ and so $P u=z$.

Therefore $z=P u=S u$ and since the pair $(P, S)$ are compatible of type $(A-1)$, by proposition 3.2 we have $P S u=S S u$, i.e. $P z=S z$. Hence

$$
\begin{aligned}
& {[1+p \cdot M(S z, T z, k t)]^{*} M(P z, Q z, k t)} \\
& \geq p\left[M(P z, S z, k t)^{*} M(Q z, T z, k t)+M(P z, T z, k t)^{*} M(Q z, S z, k t)\right]+ \\
& M(S z, T z, t)^{*} M(P z, S z, t)^{*} M(p z, S z, t)^{*} M(p z, T z, t) M(Q z, S z, t)
\end{aligned}
$$

Which yields $M(p z, z, k t) \geq M(P z, z, t)^{*} 1^{*} 1^{*} M(p z, z, t)^{*} M(z, P z, t)$ and so $p z=z$. Hence $z$ is a common fixed point of $P, Q, S$ and $T$. Finally the uniqueness of $z$ as a common fixed point of $P, Q, S$ and $T$ follows easily from (4.2).

If we take $p=0$ in the Theorem (4.2) we have the following: 
Corollary 1. Let $\left(X, M,{ }^{*}\right)$ be a complete $F M$-space with $t^{*} t \geq 1, t \in[0,1]$, and let $P, Q, S$ and $T$ be self maps of $X$ satisfying (4.1), (4.4), (4.5) and

$$
\begin{gathered}
M(P x, Q y, k t) \geq M(S x, T y, t)^{*} M(P x, S x, t)^{*} \\
M(Q y, T y, t)^{*} M(P x, T y, \alpha t)^{*} M(Q y, S x,(2-\alpha) t)
\end{gathered}
$$

for all $x, y$ in $X, t>0$, and $\alpha \in(0,2)$. Then $P, Q, S$ and $T$ have a unique common fixed point in $X$.

If we take $S=T$ and $P=Q$ in Theorem 4.2, we have the following:

Corollary 2. Let $\left(X, M,^{*}\right)$ be a complete $F M$-space with $t^{*} t \geq t, t \in[0,1]$, and let $P$ and $S$ be self maps of $X$ such that the pair $(P, S)$ are compatible of type $(A-1)$ or type $(A-2)$ and $P(X) \subseteq S(X)$.

If $S$ is continuous and there exists a constant $k \in(0,1)$ such that

$$
\begin{aligned}
& {[1+p \cdot M(S x, S y, k t)]^{*} M(P x, P y, k t)} \\
& \geq p\left[M(P x, S x, k t)^{*} M(P y, S y, k t)+M(P x, S y, k t)^{*} M(P y, S x, k t)\right] \\
& +M(S x, S y, t)^{*} M(P x, S x, t)^{*} M(p y, S y, t)^{*} \\
& M(P x, S y, \alpha t)^{*} M\left(P y, S x,(2-\alpha)^{*} t\right)
\end{aligned}
$$

for all $x, y$ in $X, p \geq 0, t \geq 0$ and $\alpha \in(0,2)$. Then $P$ and $S$ have a unique common fixed point in $X$.

In [6] Grabiec presented the fuzzy version of the Banach contraction theorem as follows:

Corollary 3. Let $\left(X, M,^{*}\right)$ be a compatible $F M$-space with $t^{*} t \geq 1, t \in[0,1]$, and let $P$ be self map of $X$. If there exists a constant, $k \in(0,1)$ such that

$$
M(P x, P y, k t) \geq M(x, y, t) \text { for all } x, y \text { in } X \text { and } t \geq 0,
$$

then $P$ has a unique common fixed in $X$.

Proof. This corollary follows from our Corollary 4.4 since (4.7) with $P=S$ and $p=0$ includes (4.8).

The above fixed point theorem extend, generalize and fuzzify several fixed point theorems on metric spaces, Menger probabilistic metric spaces, uniform spaces and $F M$-spaces ([6] - [11], [13] - [15], [20] - [23]).

\section{An Application}

In this section, we apply Corollaries (4.4) and (4.5) to establish the following result on the product space.

Theorem 5.1. Let $\left(X, M,^{*}\right)$ be a complete $F M$-space with $t^{*} t \geq t, t \in[0,1]$, and let $P$ and $Q$ be two maps on the product $X \times X$ with values in $X$. If there 
exists a constant $k \in(0,1)$ such that

$[1+p \cdot M(x, u, k t)]^{*} M(P(x, y), Q(u, v), k t)$

$\left.\geq p M(P(x, y), x, k t)^{*} M(Q(u, v), u, k t)+M(P(x, y), u, k t)^{*} M(Q(u, v), x, k t)\right]$

${ }^{*} M(P(x, y), x, t)^{*} M(Q(u, v), u, t)^{*} M(x, u, t)^{*} M(y, u, t)^{*}$

$M\left(P(x, y, u, \alpha t)^{*} M(Q(u, v, x,(2-\alpha) t)\right.$

for all $x, y$ in $X, p \geq 0, t>0$ and $\alpha \in(0,2)$, then there exists exactly one point $w$ in $X$ such that $\bar{P}(w, w)=w=Q(w, w)$.

Proof. By (5.1) we have

$$
\begin{aligned}
& {[1+p \cdot M(x, u, k t)]^{*} M(p(x, y), Q(u, y), k t)} \\
& \geq p\left[M(P(x, y), x, k t)^{*} M\left(Q(u, y, u, k t)+M(p(x, y), u, k t)^{*} M(Q u, y), x, k t\right)\right] \\
& +M(p(x, y), x, t)^{*} M(Q(u, y), u, t)^{*} M(x, u, t)^{*} \\
& M(P(x, y), u, \alpha t)^{*} M(Q(u, y), x,(2-\alpha) t)
\end{aligned}
$$

for all $x, y, u$ in $X$. Therefore, by Corollary 4.4 for each $y$ in $x$, there exists one and only one $z(y)$ in $X$ such that

$$
P(z(y), y)=z(y)=Q(z(y), y) .
$$

Now for any $y, y \prime$ in $X$ by (5.1) with $\alpha=1$, we have

$$
\begin{aligned}
& {[1+p \cdot M(z(y), z(y \prime), k t)]^{*} M\left(P(z(y), y), Q\left(z\left(y^{\prime}\right), y^{\prime}\right), k t\right) } \\
\geq & p\left[M(P(z(y), y), z(y), k t)^{*} M\left(Q\left(z(y \prime), y^{\prime}\right), z\left(y^{\prime}\right), k t\right)+M\left(P(z(y), y), z\left(y^{\prime}\right), k t\right)\right. \\
& \left.{ }^{*} M(Q(z(y \prime), y \prime), z(y), k t)\right]+M(P(z(y), y), z(y), t)^{*} M\left(Q\left(z\left(y^{\prime}\right), y^{\prime}\right), z\left(y^{\prime}\right), t\right) \\
& { }^{*} M\left(z(y), z\left(y^{\prime}\right), t\right)^{*} M\left(y, y^{\prime}, t\right)^{*} M\left(P(z(y), y), z\left(y^{\prime}\right), t\right)^{*} M\left(Q\left(z\left(y^{\prime}\right), y^{\prime}\right), z(y), t\right)
\end{aligned}
$$

i.e.

$$
\begin{aligned}
& M(z(y), z(y \prime), k t) \\
& \geq 1^{*} 1^{*} M(z(y), z(y \prime), t)^{*} M(y, y \prime, t)^{*} M(z(y), z(y \prime), t)^{*} M(z(y \prime), z(y), t)
\end{aligned}
$$

i.e.

$$
\begin{aligned}
M(z(y), z(y \prime), k t) & \geq M(z(y), z(y \prime), t)^{*} M(y, y \prime, t) \\
& \geq M\left(z(y), z\left(y^{\prime}\right), t / k_{n}\right)^{*} M(y, y \prime . t) \\
& \rightarrow M(y, y \prime, t)
\end{aligned}
$$

Therefore, Corollary 4.5 yields that the map $z($.$) of X$ into itself has exactly one fixed point $w$ in $X$, i.e. $z(w)=z$. Hence by $(5.2) w=z(w)=P(w, w)=$ $Q(w, w)$. This completes the proof. 


\section{REFERENCES}

[1] R. Badard, Fixed Point Theorems for fuzzy numbers, Fuzzy sets and systems, 13(1984) 291-302.

[2] S. Banach, Theoric les Operations Lineaires, manograie Mathematyczne, Warsaw, 1932.

[3] D. Butnariu, Fixed points for fuzzy mappings, Fuzzy Sets and Systems, 7 (1982) 191207.

[4] M. Edelstein, On fixed and periodic points under contraction mappings, J. London Math. Soc., 37 (1962) 74-79.

[5] J. X. Fang, On fixed point theorems in fuzzy metric spaces, Fuzzy Sets and Systems, (1992) 107-113.

[6] M. Grabiec, Fixed points in fuzzy metric space, Fuzzy Sets and Systems, 27 (1988) 385-389.

[7] K. Iseki, Some applications of banach type construction principles, Math. Sem .Notes, Kobe Univ., 4 (1976) 211-214.

[8] I. Istratescu, A fixed point theorem for mappings with a probabilistic contractive iterate, Rev. Roum. Math. Purest Appl., 26 (1981) 431-435.

[9] G. Jungck, Commuting mappings and fixed points, Amer.Math, Monthly, 83 (1976) 261-263.

[10] G. Jungck, Compatible mappings and common fixed points, Internat. J. Math. and Math.Sci., 9 (1986) 771-779.

[11] G. Jungck, Common fixed points for commuting and compatible maps on compacta, Proc. Amer. Math. Soc., 103 (1988) 977-983.

[12] I. Kramosil and J. Michalek, Fuzzy metric and statistical metric spaces, Kybemetica, 11 (1975) 336-344.

[13] S. N. Mishra, N. Sharma and S. L. Singh, Common fixed points of maps on fuzzy metric spaces, Internat. J. Math. and Math. Sci., 17 (1994), 253-258.

[14] B. E. Rhoades, A comparison of various definitions of contractive mappings, Trans. Amer.Math. Soc., 226 (1977) 257 -290.

[15] B. E. Rhoades, Contractive definitions revisited, Contemporary Mathematics ,21 (1983) 189-205.

[16] B. Schweizer and Asklar, Statistical metric spaces, Pacific J. Math, 10 (1960) 313-334.

[17] V. M. Sehgal and A.T. Bharucha-Reid, Fixed points of contraction mappings on probabilistic metric spaces, Math Systems Theory, 6 (1972) 97-102.

[18] S. Sessa, On a weak commutativity condition of mappings in fixed point considerations, Publ. Inst. Math (Beograd), 32(46) (1982) 149-153.

[19] A. P. Shostak, Two decades of fuzzy topology: basic ideas, notions and results, Russian Math. Surveys, 44:6 (1989) 123-186.

[20] S. L. Singh, Some common fixed point theorems in L-spaces, Math.Sem. Notes, Kobe Univ., 7 (1979) 91-97.

[21] S. L. Singh and S. Kasahara, On some recent results on fixed common fixed points, Indian J. Pure Appl. Math., 13 (1982) 757 -7 61; Corrigendum 14 (1983) 1075.

[22] S. L. Singh and B.Ram, Common fixed points of commuting mappings in 2-metric spaces, Math. Sem. Notes. Kobe Univ., 10 (1982) 197-208.

[23] B. M. L. Tivari and S. L. Singh, A note on recent generalizations of Jungck Contraction Principle, J. UPGC Soc., (1986) 13-18.

[24] M. D. Weiss, Fixed points separation and induced topologies for fuzzy sets, J. Math. Anal. Appl ., 50 (1975) 142-150.

[25] L. A. Zadeh, Fuzzy sets, Inform.Control, 8 (1965), 338-353.

Received: May 19, 2006 